\title{
PrevalÊnCIA de Sintomas respiratóRIOS E CONDIÇÕES CLÍNICAS ASSOCIADAS À ASMA EM ESCOLARES DE 6 A 14 ANOS NO RIO DE JANEIRO
}

\author{
Andréa L. Guimaräes Teldeschi, Clemax Couto Sant'anna*, Vera Lúcia T. Aires \\ Trabalho realizado no Departamento de Pediatria da Faculdade de Medicina da Universidade Federal do Rio de Janeiro
}

RESUMO-OB]Etrvo.Avaliaraprevalênciadesintomas respiratóriosecondições clínicas associadasàasmabrônquicae broncoespasmo induzido por exercício (BIE) em duas populações de escolares de instituições públicas e privadas.

Método. Realizadoinquéritotransversalem 294 I escolares de6a I4anos, sendo I59| da rede municipal pública(RPb) e |350 da rede particular (RPt), matriculadosnas classes dealfabetizaçãoeprimeirograude escolas dazonaoeste do Rio de Janeiro. Paraas entrevistasfoi empregado oformulário "ATS-DLD78-C"modificado.

RESULTADOs. Segundo a sequêencia de perguntas do formulário, houve referência a chiado em 842 (28,6\%) entrevistados; BIE em 684 (23,3\%); internaçōes hospitalares por problemas respiratórios em 342 (II,6\%); uso préviodebroncodilatadores (BD)em 1072(36,5\%); bronquioliteem 40(1,4\%); pneumonia em 185 (6,3\%); diagnóstico médico de asma brônquica em 779 (26,5\%); riniteem 1088(37\%);atopiafamiliar em I794 (61\%)eimunoterapia em $577(19,6 \%)$. A comparação estatistica entre os dois grupos de escolas mostroumaior freqüênciade BIE, hospitalizaçãoe epneumoniana $\mathrm{RPb}(26,3 \%$, $15,9 \%$ e $7,2 \%$, respectivamente). NaRPtfoimaior afreqüência de história familiardeatopiaeempregodeimunoterapia $(63,7 \%$ e $23,6 \%$, respectivamente). Aplicando-se a pontuação estabelecida pel oformulário, o diagnóstico de asma ocorreuem 638 (21,7\%) escolares, sendo 55\% da RPb e $45 \%$ da RPt. Adistribuição dos casos deasmapelas escolas evidenciouque $354(22 \%)$ eram daRPbe 284 (21\%) daRPt(n.s.).

ConcLusÃ̈o. A prevalência de asmafoielevadaentre escolares da RPte da RPb, sem diferença estatística entreambas. Os quadros de BIE também foram muito prevalentes naamostraestudada, com maior freqüência naRPb.

Unitermos: Sintomas respiratórios. Asma. Broncoespasmo induzido por exercício. Criança. Adolescente asma. Sinais e sintomas.

\section{INTRODUÇã̃o}

As doenças respiratórias caracterizadas por espirros, coriza, prurido, obstrução nasal, tosse, dispnéia e hipersecreção brônquica, freqüentes na infância, têm aumentado sua prevalência nas últimas duas décadas, possivelmente por irritação brônquica de causas infecciosas e não infecciosas, como a fumaça de cigarro e poluentes atmosféricos, tão comuns em nossos dias no ambiente urbano'.

Aasma é conceituada como uma doença inflamatória, na maioria dos casos acompanhada de hiperresponsividade brônquica, e

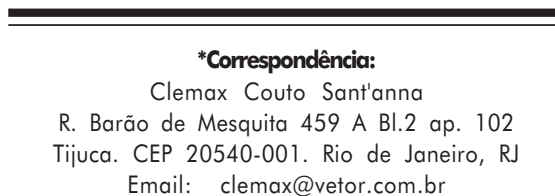

caracterizada por resposta exagerada das vias aéreas a vários estímulos físicos, químicos ou farmacológicos, com limitação do fluxo aéreo. É considerada uma doença paroxística, que se manifesta por sibilos mais audíveis na fase expiratória, associados a grande quantidade de secreção de muco. Durante o período não paroxístico pode se manifestar por tosse noturna ou após atividadesfísicas ${ }^{2}$. Seu conceito vem se tornando mais complexo e atualmente considera-se uma doença na qual interagem determinantes genéticos e ambientais. É uma condição comum que tem impacto sobre a qualidade de vida e na demanda do Sistema de Saúde. Hátendência mundial de aumento da sua morbidade, mortalidade e hospitalização nafaixa etária pediátrica ${ }^{3,4}$. No Brasil, Rosário
Filho ${ }^{5}$ relatou aumento da venda de medicamentos para tratamento de asma e de internações hospitalares pela doença.

Questionários que abordam sintomas respiratórios parecem ser os melhores instrumentos de pesquisa para estudo de prevalência de asma em grandes populações ${ }^{6}$. No entanto, observa-se ampla variação na prevalência da doença, que pode ser explicada pela falta de uniformidade no conceito médico de asma, gerando diferentes interpretações dos resultados?.

Uma vez que a definição de asma se modificou com o tempo, os inquéritos que se baseiam no diagnóstico clínico podem refletir apenasaterminologia empregada pelos médicos de cada localidade e não a prevalência real da doença. Por outro lado, aasma, além da sua 
manifestaçãa típica de sibilância, adota outras formas, como dor torácica, tosse seca crônica do tipo irritativa ou sintomas respiratórios após exercício. Além disso, poucos trabalhos na literatura abordam o relato do quadro clínico sugestivo de broncoespasmo induzido por exerćício (BIE) que não é pesquisado de forma rotineira nos trabalhos de epidemiologia da asma. ${ }^{8,9}$

A prática de inquéritos amplos naárea de Saúde deve ser estimulada em nosso país, na qual o nível social deve ser considerado na análise do processo saúde-doença. Portanto, no caso da asma, torna-se útil a padronização de formulário simples para aplicação na comunidade, a fim de sugerir medidas preventivas para diminuir sua morbidade ${ }^{3}$.

O objetivo do trabalho foi contribuirpara o conhecimento dos problemas respiratórios, especialmente a prevalência de asma e de broncoespasmo induzido por exercício (BIE) em estudantes de escolas públicas e particulares da zona oeste do Rio de Janeiro. Não se encontrou nenhum estudo epidemiológico prévio semelhante a este na região escolhida, na qual há grande concentração de crianças e adolescentes, elevada umidade relativa do ar e freqüentes variações climáticas

\section{Métodos}

Este trabalho é um inquérito transversal, realizado prospectivamente, no período de setembro a novembro de 1995, com a distribuição de questionários a escolares das classes de alfabetização e do primeiro grau, matriculados em nove escolas da rede pública municipal (RPb) e em sete da rede particular (RPt), localizados nas XVI e XXIV Regiões Administrativas (R.A) do município do Rio de Janeiro que compreende os bairros da Barra da Tijuca, Recreio dos Bandeirantes e lacarepaguá.

A realização da pesquisa foi autorizada pelos diretores das escolas da Rpt e pelo Distrito Escolar (DEC), no caso da RPb. As

\begin{tabular}{l}
\hline \multicolumn{2}{c}{ Anexo I - Formulário } \\
\hline IDENTIFICAÇÃO DA CRIANÇA \\
NOME: \\
SEXO: \\
DATA DE NASCIMENTO: \\
ENDEREÇO:
\end{tabular}

\section{Qual o seu grau de parentesco com a criança?}

( ) MÃE ( ) PAI ( ) TIOS ( )AVÓS ( )OUTROS

I) Acriançajáteve episódio de chiado que causoufalta de ar oufôlego curto? ( ) SIM ( ) NÃO

2) Acriançajáteve episódio de tosse, cansaço, chiado, dor no peito ouapenasfatta de arapósjogos ouexerćícios?
( ) SIM
( ) NÃO

3) Acriançajáfoi hospitalizada por doença pulmonar grave ou com catarro no peito? ( ) SIM ( ) NÃO

4) Acriançajáfez uso de alguma das seguintes medicações por mais detrêsocasiões?
( ) AEROLIN
( ) AEROPLUX
( )AMINOFILINA
( ) BEROTEC
( ) ATROVENT
( ) ADRENALINA
( ) TEOFILINA
( ) BRICANYL
( ) SALBUTAMOL

5) Acriançaapresentoualgumadasseguintes doenças?
( ) bronquiolite
( ) bronquite
( ) bronquite asmática
( ) pneumonia
( ) asma

6) Omédicoalgumavez disse que seufilhotemasma, bronquite asmática oubronquite alérgica?
( ) SIM
( ) NÃO

7) Acriança costuma apresentar comfrequiênciaespirros, coceira no nariz, entupimento nasal ou coriza?
( ) SIIM
( ) NÃO

8) Nafamilia há casos de bronquite, asma oueczema?

$\begin{array}{ll}\text { ( )avóspaternos } & (\text { )avósmaternos } \\ (\text { ) pai } & (\text { mãe } \\ \text { ( )tios paternos } & (\text { )tiosmaternos } \\ \text { ( )irmãos } & (\text { )outros,especificar: }\end{array}$

9) Acriançausa oujáusouvacinas paraalergia?
( ) SIM
( ) NÃO

famílias dos escolares foram esclarecidas quanto ao teor do trabalho pela pesquisadora principal e pelos professores das classes envolvidas.

O instrumento para coleta dos dados foi modificado de Esteves, Solé e Ferraz ${ }^{9}$ para melhor compreensão por parte do responsável, considerando que não haveria entrevistador, sendo porém mantidos o mesmo número de questões e a mesma pontuação, ou seja, o mesmo "ponto de corte" para diagnóstico de asma. Foi considerado como asma a pontuação igual ou superior a sete (anexos | e 2).

O número amostral foi baseado em dois trabalhos nacionais de estudo de prevalênciade asma citados por Fritscher et al. 10: um com prevalência de 16,5\%, em escolares de 10-18 


\begin{tabular}{ccc}
\hline \multicolumn{3}{c}{ Anexo 2 - Pontuação do formulário } \\
\hline QUESTÕES & VARIÁVEIS & PONTOS \\
\hline 1 & Chiado & $\mid$ \\
2 & Exercício & 2 \\
3 & Hospitalização & $\mid$ \\
4 & Broncodilatadores & $\mid$ \\
5 & Bronquiolite, asma ou & 1 \\
& Pneumonia & \\
6 & Diagnósticodeasma & 2 \\
7 & Sintomas derinite & 1 \\
8 & Atopiafamiliar & 1 \\
9 & Imunoterapia & $\mid$ \\
\hline
\end{tabular}

anos de idade e o outro com 19,1\%, em crianças de até cinco anos de idade. Considerando uma precisão de 1,5\% e uma prevalência elevada da ordem de 20\%, seria necessário uma amostra de 273 I escolares; sendo assim, a amostra colhida foi superior à estimativa de prevalência mais elevada.

Os dados foram coletados e analisados em computador através do banco de dados Epinfo 5. O teste do "qui quadrado" foi utillizado para determinarasignificânciaestatísticanas differentes prevalências. Foi considerado como significativo o valor do p menor que 0,05 .

\section{Resultados}

No total, foram distribuídos 7000 questionários. Dos 3000 da RPt foram devolvidos 1389, o que corresponde a um índice de devolução de 46,30\%. Dos 4000 da RPb, foram devolvidos 1726 (43, I 5\%). Houve diferença estatística, evidenciando maior devolução na RPt. Dos 3 I I 5 devolvidos, ainda foram excluídos 174 por erro de preenchimento, perfazendo o total de 294 I formulários válidos, dos quais 2338 (79,5\%) tinham sintomas respiratórios e $603(20,5 \%)$ negavam tais sintomas.

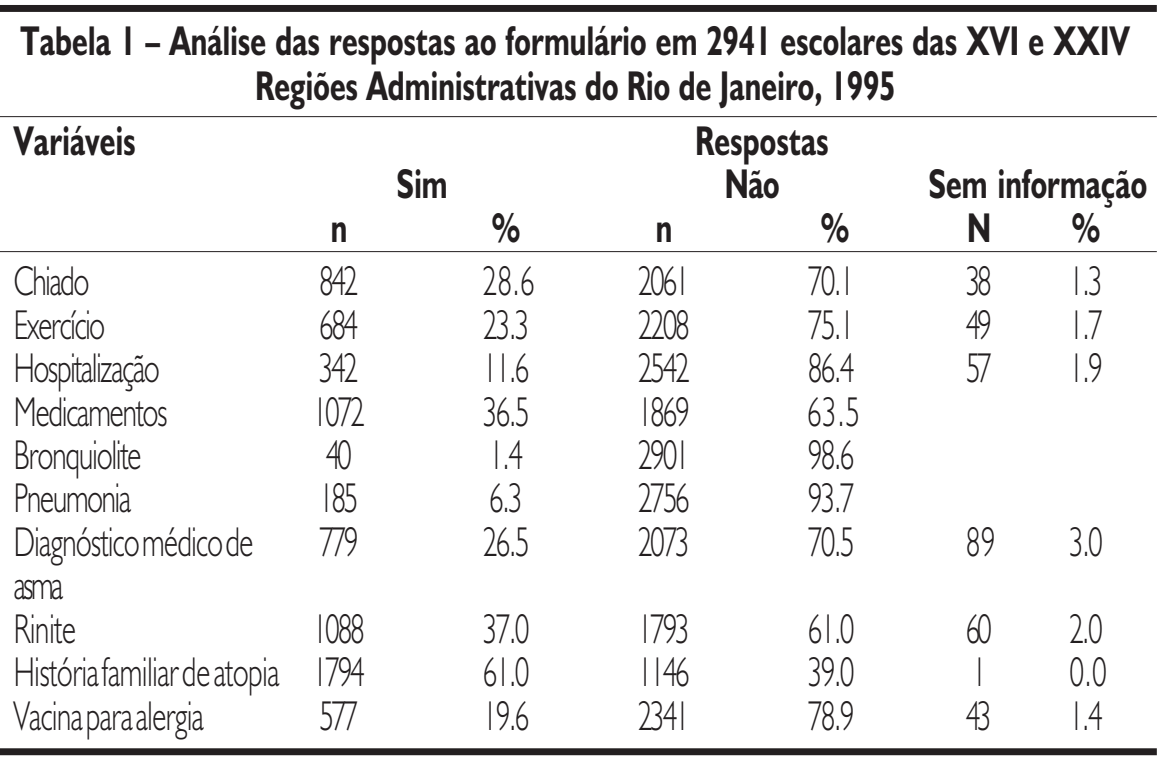

Segundo o IplanRio de 1995, na rede particular naquele ano foram matriculadas 35222 crianças da classe de affabetização à oitava série do primeirograu. Logo, aamostra estudada de 1350 crianças, corresponde a 3,83\% deste segmento de escolares. $\mathrm{Na}$ rede municipal foram estudados $|59|$ | escolares, o que corresponde a 2,8\% dos 56948 matriculados.

A Tabela I analisa as variáveis incluídas no formulário e suas respectivas prevalências.

$\mathrm{Na}$ Tabela 2 compara-se a prevalência das 10 variáveis do formulário entre os escolares da RPb e os da RPt. Tal comparação mostrou maior prevalência na RPb de sintomas sugestivos de BIE, internação hospitalar por problemas respiratórios e pneumonia. $\mathrm{Na}$ RPt foi maior a ocorrência de história familiar de bronquite, asma ou eczema e de uso de vacinas para alergia (imunoterapia).

Procedendo-se a análise baseada na pontuação dos questionários, estabeleceuse o diagnóstico de asma naqueles que alcançaram sete ou mais pontos. Foi possível, portanto, identificar 638/294I escolares com asma, representando $21,7 \%$ da amostra. Éimportante ressaltar, porém, que
603/294 lescolares, isto é, 20,5\% obtiveram zero de pontuação, o que significa que as respostas foram todas negativas.

A Tabela 3 mostra o comportamento de asmáticos nos dois grupos de escolares em relação às variáveis incluídas no formulário.

Estudando-se a distribuição dos 638 casos de asma entre as escolas de ambas as redes, constatou-se que 354/I59 I eram da RPb e 284/I350, da RPt, correspondendo a percentuais de $22 \%$ e $21 \%$, respectivamente $\left(X^{2}=0,56 ; p=0,45\right.$ ates cor.).

Ainda baseado na análise do formulário, foram encontrados 684/2941 (23,3\%) estudantes com BIE, assim considerados, pois seus responsáveis responderam afirmativamente à segunda questão do formulário, que consistia em aferir se a criança já apresentara história de tosse, cansaço, chiado, dor no peito oufalta de ar após jogos ou exercícios.

A análise dos 684 casos de BIE foi feita em relação aos 294 I escolares da amostra, buscando-se sua relação com a asma. Dos 638 asmáticos, 443 ( 69,4\%) também tinham BIE e dos 2303 não asmáticos, 24I $(10,5 \%)$ tinham BIE $\left(X^{2}=970,7 ; p<\right.$ $0,00001)$ 


\section{Tabela 2 - Análise comparativa das variáveis incluídas no formulário entre 294I escolares da rede pública e particular - XVI e XXIV Regiōes Administrativas do Rio de Janeiro, 1995}

\begin{tabular}{|c|c|c|c|c|c|c|}
\hline Variáveis & $\begin{array}{c}\text { Públicas } \\
\text { n }\end{array}$ & $\begin{array}{l}\text { Escolas } \\
(\mathrm{n}=|59|) \\
\%\end{array}$ & $\begin{array}{c}\text { Particulares } \\
n\end{array}$ & $\begin{array}{c}(n=1350) \\
\%\end{array}$ & $\mathrm{X}^{2}$ & Valor $P$ \\
\hline $\begin{array}{l}\text { Chiado } \\
\text { Exerćíio } \\
\text { Hospitalização } \\
\text { Medicamentos } \\
\text { Bronquilite } \\
\text { Pneumonia } \\
\text { Diagnósticomédico de asma } \\
\text { Rinite } \\
\text { Históriafamillardeatopia } \\
\text { Vacinas paraalergia }\end{array}$ & $\begin{array}{l}474 \\
419 \\
253 \\
580 \\
21 \\
115 \\
411 \\
589 \\
934 \\
258\end{array}$ & $\begin{array}{l}29.8 \\
26.3 \\
15.9 \\
36.5 \\
1.3 \\
7.2 \\
25.8 \\
37.0 \\
58.7 \\
16.2\end{array}$ & $\begin{array}{r}368 \\
265 \\
89 \\
492 \\
19 \\
70 \\
368 \\
499 \\
860 \\
319\end{array}$ & $\begin{array}{r}27.3 \\
19.6 \\
6.6 \\
36.4 \\
1.4 \\
5.2 \\
27.3 \\
7.0 \\
63.7 \\
23.6\end{array}$ & $\begin{array}{c}2.17 \\
18.03 \\
60.69 \\
0.00 \\
0.00 \\
4.83 \\
0.69 \\
0.00 \\
7.46 \\
24.98\end{array}$ & $\begin{array}{l}0.14 \\
0.0000 \\
0.0000 \\
0.97 \\
0.96 \\
0.02 \\
0.40 \\
0.99 \\
0.006 \\
0.0000\end{array}$ \\
\hline
\end{tabular}

Tabela 3 - Análise das variáveis incluídas no formulário entre 638 escolares com asma

\begin{tabular}{|c|c|c|c|c|c|c|}
\hline Variáveis & $\begin{array}{c}\text { Públicas } \\
\mathbf{N}\end{array}$ & $\begin{array}{c}\text { Escolas } \\
n=354 \\
\%\end{array}$ & $\begin{array}{c}\text { Particulares } \\
\mathrm{n}\end{array}$ & $\begin{array}{c}n=284 \\
\%\end{array}$ & $X^{2}$ & Valor $\mathbf{P}$ \\
\hline $\begin{array}{l}\text { Chiado } \\
\text { Exerćcicio } \\
\text { Hospitalização } \\
\text { Medicamentos } \\
\text { Bronquiolite } \\
\text { Pneumonia } \\
\text { Diagnóstico médicode asma } \\
\text { Rinite } \\
\text { Históriafamiliarde atopia } \\
\text { Vacinasparaalergia }\end{array}$ & $\begin{array}{l}298 \\
264 \\
159 \\
328 \\
17 \\
86 \\
317 \\
274 \\
315 \\
158\end{array}$ & $\begin{array}{l}84.2 \\
74.6 \\
44.9 \\
92.7 \\
4.8 \\
24.3 \\
89.5 \\
77.4 \\
89.0 \\
44.6\end{array}$ & $\begin{array}{l}255 \\
179 \\
53 \\
275 \\
13 \\
56 \\
269 \\
239 \\
254 \\
153\end{array}$ & $\begin{array}{l}89.8 \\
63.0 \\
18.7 \\
96.8 \\
4.6 \\
19.7 \\
94.7 \\
84.2 \\
89.4 \\
53.9\end{array}$ & $\begin{array}{l}3.82 \\
9.37 \\
47.78 \\
4.52 \\
0.00 \\
1.65 \\
4.96 \\
4.14 \\
0.00 \\
5.02\end{array}$ & $\begin{array}{l}0.05 \\
0.00 \\
0.00 \\
0.03 \\
0.95 \\
0.19 \\
0.02 \\
0.04 \\
0.95 \\
0.02\end{array}$ \\
\hline
\end{tabular}

\section{Dıscussão}

Para o emprego de questionários de sintomas respiratórios em crianças, visando excluir o entrevistador, foi necessário escoIher formulário que abordasse os sintomas de forma simples e permitisse, através de um sistema de pontuação, o diagnóstico de asma, guardando boa correlação com o diagnóstico médico. Neste sentido, adotamos o questionário de Esteves, Solé e
Ferraz ${ }^{9}$ queem Embú, São Paulo, simplificaram e validaram o documento "ATS-DLD-78-C" para diagnóstico de asma em levantamentos populacionais. Foram selecionadas nove questões e a cada uma delas atribuída uma pontuação. Esse questionário constitui-se um bom instrumento para levantamentos populacionais de asma na infância, mostrando alta sensibilidade, especificidade e reprodutibilidade, com ponto de corte de sete pontos.
Através de questionários, é possível a realização de grandes inquéritos populacionais, porém o método tem restrições, cuja maior delas é a dificuldade de retorno dos formulários. Apesar de todas as limitações de estudos dessa natureza em nosso meio, alcançou-se um percentual de devolução em torno de $45 \%$ que atendeu ao número amostral previsto para o trabalho. Os índices de devolução são variados mesmo em países desenvolvidos. Clifford etal. . em Southampton, Inglaterra, obtiveram uma devolução de $84 \%$, enquanto Boezen et al. ", na Holanda, obtiveram 56\% eWhincup etal. ${ }^{12}$, na Inglaterra e no País de Gales, 42\%. No presente trabalho, foi encontrada prevalência de história de chiado, causando falta de ar ou fôlego curto, em 28,6\% dos escolares. Não se observou diferença entre os escolares da RPb e os da RPt. No entanto, a prevalência dessa mesma variável foi maior entre os 638 asmáticos se comparada à amostra total estudada, constituindo-se, pois, em viés de seleção, uma vez que tais queixas são características da asma.

Na literatura a prevalência de história de sibilos (chiado) évariada pelos diversos continentes, com percentuais entre $9 \%$ a $25 \%$, o que seria explicado por condições climáticas distintas, associadas às diferentes características genéticas das populações ${ }^{13-20}$.

Em nossa amostra, a prevalência de BIE foi superior a 19\%, com valores mais elevados entre os escolares da RPb. Pode-se especular, dentre outras causas, que haja baixo condicionamento físico entre os escolares da RPb. Existem poucos dados na literatura que avaliem o quadro clínico de BIE, para que se possa comparar os diferentes países. A prevalência mais baixa, emtorno de 5\% foi descrita na Suécia e na Suíça, enquanto que a mais elevada, alcançou 20,4\% em adolescentes chilenos $8,16,17,21,22$.

Encontrou-se prevalência de 11,6\% de internações hospitalares por doença pulmonar grave ou com "catarro no peito". A 
Teldeschi ALG et AL.

freqüência foi significativamente maior em escolares da RPb. Por sua vez, considerando apenas os 638 escolares com asma, a ocorrência de internações hospitalares alcançou $33 \%$, percentual bem maior que da amostra geral. Além disso, também foi significativamente maior nos escolares asmáticos da RPb.

Apesar de a maior prevalência de internação hospitalar por problemas respiratórios entre os escolares da RPb, a prevalência de asma nos dois grupos de escolas foi semelhante, sem diferença estatisticamente significativa. Talvez, o possível desnível dos extratos socioeconômicos dos escolares seja um fator importante neste achado.

Em Southampton, Inglaterra, a prevalência de hospitalização por problemas respiratórios aos sete anos, no sexo feminino, foi de $4,5 \%$ e no sexo masculino 6,9\%. Aos II anos, os percentuais foram $3 \%$ e $4,8 \%$, respectivamente?

Quanto ao uso de broncodilatadores, não se encontrou diferença significativa entre os dois grupos de escolas, evidenciando seu amplo uso nessa amostra e refletindo os elevados percentuais de ocorrência asma que serão discutidos adiante. Em nosso estudo, das 842 crianças com relato de chiado e falta de ar, $70 \%$ referiram diagnóstico médico de asma e 79\% de uso prévio de broncodilatadores. Na literatura, a prevalência do uso de broncodilatadores em escolares, através de questionários, variou de $83 \%$ na Austrália a 46\% da Suíça e 47\% no Chile e estaria relacionada à prevalência desigual de asma nesses países' ${ }^{16}$.

A prevalência de pneumoniafoi maior nos escolares da RPb do que na RPt, supostamente porque aqueles teriam menos acesso ao sistema de saúde do que os da RPt. Por outro lado, ao se analisar apenas os asmáticos, a prevalência de pneumoniafoiigualmente elevada nos dois grupos de escolares, se comparadaàamostrageral. Contudo, a prevalência de bronquiolite ou pneumonia entre os escolares asmáticos das diferentes escolas não mostrou diferença estatística.
No presente trabalho, a freqüência do diagnóstico médico de asma foi de $26,5 \%$, embora pela pontuação do formulário os casos de asma correspondam a 21,6\%. A prevalência da asma varia de quase zero entre os esquimós a valores bem elevados em países como o Chile, Austrália e Nova Zelândia. ${ }^{15-17}$

O baixo nível socioeconômico tem sido associadoa maior prevalência de asma. Neste trabalho, no qual seria possível supor que os escolares da RPb fossem mais carentes que os da RPt, não houve diferença significativa entre os grupos, sendo elevada a prevalência de asma em ambos. Sennhauser e Kuhni, na Suíça, atribuíram à baixa prevalência da asma encontrada em seu estudo, muito inferior à observada no hemisfério sul, ao alto nível socioeconômico da população estudada. Entretanto, os autores assinalam que a razão de tal diferença entre os dois hemisférios ainda é desconhecida. As áreas de maior prevalência se localizam abaixo do trópico de Capricórnio, em regiões costeiras, com maior umidade e grande variabilidade detemperatura ou microclimas. ${ }^{20,21}$

No Brasil, na cidade de Cascavel, a prevalência de asma na populaçãão geral foi de $12 \%$, porém dos 6 aos 10 anos alcançou 22,6\%. ${ }^{3}$ Em Fernando de Noronha, ilha praticamente isenta de poluição, foi de II,2\% em menores de 6 anos $^{23}$, e em Ribeirão Preto-SP, a prevalência foi de $3,4 \%$, no sexo feminino e $2,4 \%$ no masculino ${ }^{24}$, e em Porto Alegre, RS em 1989 de $16,5 \%$ em adolescentes de 10 e 18 anos. ${ }^{10}$

Quanto à rinite, nossos dados mostram que não houve diferença de prevalência entre os estudantes da RPb e da RPt. Porém, considerando somente as crianças asmáticas, houve maior freqüência na $\mathrm{RPb}$, bem como predomínio do sexo masculino na faixa etária dos 6 aos 8 anos e do sexo feminino dos 12 aos 14 anos, neste último caso, à semelhança de Sennhauser e Kuhnil ${ }^{21}$. Além disso, 47 \% das crianças com sintomas sugestivos de rinite apresentavam asma, o que mostra a grande freqüência desta associação, como em outros estudos. ${ }^{25,26}$

Observamos que 19,6\% dos escolares relataram ter usado vacinas para alergia. A mesma prática foi citada por $48,7 \%$ das crianças asmáticas. Houve diferença significativa no uso de imunoterapia entre os dois tipos de escolas, com maior freqüência entre os alunos da RPt, cujas famílias também tinham prevalência significativamente maior de história familiar de atopia. Esta constatação poderia também estar relacionada à questão do poder aquisitivo, pois poucos serviços públicos de saúde fornecem imunoterapia gratuitamente.

Concluindo, destacamos que questionários que abordam sintomas respiratórios são instrumentos de valor em levantamentos populacionais para determinação da prevalência de problemas respiratórios em geral, e da asma, em particular. ${ }^{27}$ Encontrouse elevada prevalência de asma entre os escolares de ambas as redes de escolas, sem diferença estatística. Os quadros de BIEtambém foram muito prevalentes, sendo que na RPb houve maior número de casos.

Este trabalho teve o apoio financeiro do CNPq.

\section{SUMMARY}

\section{Prevalence OF ResPiRatory syNPTOMS AND ASTHMA IN SCHOOLCHILDREN IN RIO DE JANEIRO}

OBEETTVE. We compared the prevalence of respiratory symptoms and clinical conditions associated to bronchial asthma and exerciseinduced bronchospasm (EIB) in schoolchildren from public and private schools.

Methods. We conducted a crosssectional questionnaire (ATS-DLD-78 C modified) survey in 294I children 6-14 years of age attending public $(n=1951)$ and private $(n=1350)$ elementary and secondary schools in Rio de Janeiro. 
RESULTS. Wheezing was found in 842 (28.6\%) students; 684 students (23.3\%) showed symptoms of EIB; 342 (11.6\%) children related hospitalization owing to respiratory problems; previous use of bronchodilators (BD) was reported by 1072 (36.5\%) children; bronchiolitis, pneumonia and rhinitis were observed in respectively 40 (1.4\%), 185 (6.3\%) and 1088 (37\%) children. Family history of atopy and immunotherapy were related by 1794 (6/\%) and 577 (19.6\%) students, respectively. Asthma diagnosed by a physician was observed in 779 (26.5\%) children. Usingquestionnaire criteria we found 638 (21.7\%) children with asthma; 354 in public and 284 in private schools. Data statistical analysis showed a higher frequency of EIB, hospitalization and pneumonia in students attending public schools (26.3\%, $15.9 \%$ and $7.2 \%$, respectively). Children in private schools showed a higher frequency of family history of atopy and immunotherapy (63.7\% and $23.6 \%$, respectively).

CONCLUSIONS. We found a high prevalence of asthma among students of public and private elementary and secondary schools in Rio de Janeiro, without statistical differences between the two groups of children. Episodes of EIB were also highly prevalent in this study, with a higher frequency among students of public schools. [Rev Assoc Med Bras 2002; 48(I): 54-9]

Keywords: Respiratory symptoms. Asthma. Exercise-induced bronchospasm. Child. Adolescent.

\section{REFERÊNCIAS}

I. Whincup PH, Cook DG, Strachan DP, Strachan DP, Papacosta O. Time trends in respiratory symptoms in childhood over a 24 year period. Arch Dis Child 1993; 68:729-34.

2. Kaufman HS, Frick OL. The development of allergy in infants of allergic parents: A prospective study concerning the role of heredity. Ann Allergy 1976; 37:410-5
3. Campos HS, Machado JL, Gonfieri JH. Sintomas respiratórios, bronquite crônica e asma na população urbana de Cascavel, PR. Resultados da aplicação de um questionário padronizado numa amostra da população, em 1989. Pulmão RJ 1994; 4:|4-22.

4. Gergen PJ, Weiss KB. Changing patterns of asthma hospitalization among children: 1979-1987. JAMA 1990; 264:1688-92.

5. Rosário Fo. NA. Broncodilatadores na asma: contornando controvérsias. J Pediatr 1996; 72: $281-286$.

6. Souza RB. Questionário padronizado sobre sintomas respiratórios [dissertação]. Rio de Janeiro: Faculdade de Medicina, Universidade Federal do Rio de Janeiro; 1986

7. Clifford RD, Radford M, Howell JB, Holgate ST. Prevalence of respiratory symptons among 7 and II years old schoolchildren and association with asthma. Arch. Dis. Child 1989; 64: I|18-25.

8. Bardagi S, Agudo A, Gonzalez CA, Romero PV. Prevalence of exercise-induced airway narrowing in schoolchildren from a Mediterranean town. Am Rev Respir Dis 1993; | 47: | | | 2-5.

9. Esteves AR, Solé D, Ferraz MB. Validação do questionário "ATS-DLD-78-C" modificado para diagnóstico de asma em crianças de 0 a 13 anos. In: V Congresso Brasileiro de Alergia e Imunologia em Pediatria, II Congresso Panamericano de Alergia e Imunologia em Pediatria, III International Pediatric Consensus on the management of Asthma" e "I Congresso of the International Pediatric Allergy and Respiratory Federation, 1995, São Paulo. Anais.

10. Fritscher CC, Severo RD, Fagondes SC. Modificações na prevalência de asma brônquica em escolares de Porto Alegre. J Pneumol 1994; 20:6-10

II. Boezen HM, Schouten JP, Postmaa DS, Rijcken B. Relation between respiratory symptoms, pulmonary function and peak flow variability in adults. Thorax 1995:50:121-6.

12. Whincup PH, Cook DG, Strachan DP, Papacosta $O$. Time trends in respiratory symptoms in childhood over a 24 yaer period. Arch Dis Child 1993; 68:729-34.

13. Haby MM, Anderson SD, Peat JK, Mellis CM, Aoelle BG, Woolcock AJ. An exercise challenge protocol for epidemiological studies of asthma in children: comparison with histamine challenge. Eur Respir J 1994; 7:43-9.

14. Lee TH, Brown, MJ, Nagy L Canson R, Walpont MJ, Lay AB. Exercise-induced release of histamine and neutrophil chemotatic factor in atopic asthmatics. J. Allergy Clin Immunol 1982; 70:73.

15.Lee DA, Winslow NR, Speight ANP, Hey EN. Prevalence and spectrum of asthma in childhood. Br Med J (Clin Res Ed) 1983; 286: 1256-8.
16. Robertson CF, Bishop J, Sennhauser FH, Mallol J. International comparison of asthma prevalence in children: Australia, Switzerland, Chile. Pediatr Pulmonol 1993; 16:219-26.

17. Burr ML, Butland BK, King S, VaughamWilliams E. Changes in asthma prevalence: two surveys 15 years apart. Arch. Dis. Child 1989; 64:1452-6.

18. O'Connor GT, Dockery D, Raizenne M. Passive smoking and wheezing-related emergency room visits among children in 24 US and Canadian cities. Am Rev Respir Dis 1992; 145: A532

19. Peat JK, Toelle BG, Gray EJ, Haby MM, Belousona E, Mellis CM, et al. Prevalence and severity of childhood asthma and allergic sensitisation in seven climatic regions of New South Wales. Med J Aust 1995; 163: 22-6.

20. Flynn MGL. Respiratory symptoms, bronquial responsiveness, and atopy in Fijian and Indian children. Am J Resp Crit Care Med 1994: | 50:4|5-20.

21. Senhauser FH, Kuhni CE. Prevalence of respiratory symptom in Swiss children: is bronchial asthma really more prevalent in boys ? Pediatr Pulmonol 1995; 19:161-6.

22. Braback L, Breborowicz A, Dreborg S, Knutsson A, Pieklik H, Bjorksten B. Atopic sensitization and respiratory symptoms among Polish and Swedish school children. Clin Exp Allergy 1994; 24:826-35.

23. Sarinho ESC, Sarinho S, Ferreira OS. Fatores de risco para asma infantil em Fernando de Noronha: estudo do tipo caso-controle. J Pediatr 1995: 71:270-2.

24. Ramos MC. Sintomas respiratórios na população da cidade de Ribeirão Preto, SP (Brasil). Resultados da aplicação de um questionário padronizado Rev Saúde Publica 1983; | 7:41-52.

25. Aires VLT, Teldeschi ALG. Estudo descritivo da demanda ambulatorial de asmáticos no IPPMG-1988/1990. Arq Bras Med 1992; $66: 24-31$

26. Scadding G, Agraval A. Lower respiratory tract symptoms in peadiatric rhimitis. Eur J Allergy Clin Immunol 1995; 50:83.

27. Aguiar VAN, Beppu OS, Romaldini $H$. Validade de um questionário respiratório modificado (ATS-DLD-78) como instrumento de um estudo epidemiológico em nosso meio. J Pneumol 1988; |4:| ||-6.

Artigo recebido: 05/09/2000

Aceito para publicação: 03/07/200 I 